http://dx.doi.org/10.21611/qirt.2015.0122

\title{
Automated diagnosis of hypertension using dynamic thermography
}

Name: T.Jayanthi

Institution: Department of Biomedical Engineering, SRM University

Address: SRM University, Kattankulathur - 603203, Tamil Nadu, India

Email: jayanthi.t@ktr.srmuniv.ac.in

Contact Number: 9840490315

\section{Second Author:}

Name: M.Anburajan

Institution: Department of Biomedical Engineering, SRM University

Address: SRM University, Kattankulathur - 603203, Tamil Nadu, India

Email: hod.biomedi@ktr.srmuniv.ac.in

Introduction: World Health Organization rates hypertension as one of the most important causes of premature death worldwide. In 2025 it is estimated there will be 1.56 billion adults living with high blood pressure. Hypertension, or high blood pressure, is often called a "silent disease" as they may be no symptoms or signs. Nonetheless, it damages the body and eventually may cause problems like heart disease. The need for cost effective screening tool for the disease is the basis of this work. This study aims at detecting the vascular abnormalities using thermal imaging and brings out its potential as a tool for the evaluation of hypertension.

Method: Dynamic thermal imaging was captured by FLIR A305sc, FLIR Systems, Inc USA, infrared camera. The thermal camera was placed on a tripod at a distance of $1 \mathrm{~m}$ from the subject who is asked to sit on a fixed chair to facilitate the video recording process. The recording of the thermal infrared images was done in a room with an average room temperature of $23^{\circ} \mathrm{C}$. The camera was focused for each minute on face, left and right hand, left and right foot on both anterior and posterior regions. The video on all major regions taken for $60 \mathrm{sec}$ were stored and analyzed using FLIR research IR software. The dynamic thermal signal from all the major regions was decomposed using 1 dimensional discrete haar wavelet at level 2.The decomposition 
of the signal showed distinct peaks with less noise. Hence features like energy, power, variance, standard deviation, kurtosis and skewness were calculated from the synthesized waveform.

PC based PeriScope Device (Genesis Medical Systems, India) was used to measure the various mechanical parameters of blood flow like heart rate, systolic and diastolic blood pressure, pulse pressure, right and left brachial pulse wave velocity, carotid femoral pulse wave velocity, right and left brachial arterial stiffness index, right and left ankle arterial stiffness index, right and left ankle brachial index, aortic systolic pressure, aortic pulse pressure, diastolic pressure, aortic augmentation index and augmentation index adjusted to heart rate 75 beats/min.

Automated Classifier: The back propagation neural network (BPN) was implemented using MATLAB Version R2014a. The 2 layer network with back propagation training was used with number of hidden units being $1 / 10^{\text {th }}$ of the total input parameters [37]. The network was implemented using different combinations of input features (n) as follows:

i. Dynamic thermography features from the video signal

ii. Average temperatures from the whole body regions from static thermography

iii. Mechanical parameters of blood flow from periscope

iv. Combination of static and dynamic features

v. Combination of static, dynamic and mechanical parameters of blood flow

Results: The performance of the network was also tested by implementing input feature dimensionality reduction by PCA (SPSS Version 17.0). The BPN was implemented to diagnose the hypertensive patients. It is a two layer network where the number of hidden units was $1 / 10^{\text {th }}$ of the input features [36]. Different approaches were done with and without feature selection by PCA. The principal components that had eigen values greater than 1 were selected for the classification in each approach. When the average skin surface temperature measured using static thermography and the statistical features derived from dynamic thermography were used in BPN after feature selection by PCA, it was found that the sensitivity and specificity of the classifier for diagnosis of hypertension were found to be $92.9 \%$ and $78.6 \%$ respectively. The accuracy of network was found to be $89.7 \%$. 